\title{
RELATO DE CASO: INFARTO AGUDO DE MIOCÁRDIO EM PACIENTE EM TRATAMENTO DE PNEUMONIA POR COVID-19.
}

\section{Martina Dominick Rehn; Anibal Pereira Abelin²; Luíza Fenalte Streher ${ }^{3}$; Mônica Mokan Hasse"; Verônica Kasper5; Guilherme Stradiotto Batistella6; Adalgiso Feijó Malaguez ${ }^{7}$}

\section{RESUMO}

O objetivo desse trabalho é relatar um caso ocorrido em UTI-Covid em um hospital numa cidade central no estado do Rio Grande do SUL - Brasil referente a um paciente com 42 anos de idade com Covid-19 que teve um Infarto agudo do miocárdio (IAM), discorrendo sobre o diagnóstico e conduta. É um estudo de natureza descritiva, tipo relato de caso, em que uma consulta de artigos nas plataformas Scielo e Pubmed além de análises nas diretrizes mais recentes nacionais e internacionais de condutas para diagnóstico e tratamento para o infarto agudo do miocárdio em pacientes no período trans e pós diagnóstico de infecção com Covid-19 foi feita. Foi observado que pacientes com Covid-19 podem apresentar um estado de hipercoagulabilidade tendo como consequência o IAM. Conclui-se que com o evento de casos de Covid-19 em pessoas em adultas pode levar a Doença Arterial Coronariana.

Palavras-chave: Doença Arterial Coronariana; Hipercoagulabilidade; Complicação trombótica; coagulopatia.

Eixo Temático: Atenção Integral e Promoção à Saúde (AIPS).

\footnotetext{
${ }^{1}$ Estudante do Curso de Medicina. Universidade Franciscana. E-mail: martina.rehn@hotmail.com

2 Mestre. Docente do Curso de Medicina. Universidade Franciscana. E-mail: anibalpabelin@yahoo.com.br

${ }^{3}$ Estudante do Curso de Medicina. Universidade Franciscana. E-mail: lupifs@terra.com.br

${ }^{4}$ Estudante do Curso de Medicina. Universidade Franciscana. E-mail: monica hasse@hotmail.com

${ }^{5}$ Estudante do Curso de Medicina. Universidade Franciscana. E-mail: veerokasper@gmail.com

${ }^{6}$ Estudante do Curso de Medicina. Universidade Franciscana. E-mail: gguilhermestradiotto@gmail.com

${ }^{7}$ Orientador. Mestre. Docente do Curso de Medicina. Universidade Franciscana. E-mail: amalaguez@hotmail.com
} 


\section{INTRODUÇÃO}

Sendo uma das causas mais predominantes de morbidade e mortalidade perioperatória, a Doença Arterial Coronariana (DAC) é considerada a responsável por aproximadamente $25 \%$ do total de mortes nos países ocidentais. A sua fisiopatologia abrange fatores como a diminuição da luz da artéria, o que tem como consequência o aumento de resistência ao fluxo, a queda do suprimento sanguíneo distal à placa coronariana e também a formação de uma placa aterosclerótica no qual poderá ter maior chance de ocasionar ruptura da continuidade endotelial e formação de tampão plaquetário e trombose, podendo levar a oclusão ou suboclusão da artéria coronária e redução importante do fluxo sanguíneo coronariano e assim causar morte dos miócitos, por falta de oxigênio. Os fatores de risco para formação de placas de ateroma nas artérias são hiperlipidemia, hipertensão, diabetes, tabagismo, sexo masculino e história familiar de infarto agudo do miocárdio. (SILBERNAGL, 2016) A realização do diagnóstico é feita através do conjunto de achados clínicos, de exames eletrocardiográficos e, também, outros exames complementares. Para reduzir consideravelmente a morbidade $\mathrm{e}$ mortalidade, é preciso que seja feito seu tratamento de maneira rápida e de forma correta.

Ao avaliar a idade de pacientes com diagnóstico de IAM foi viso em um estudo de 10 anos do Framingham Heart Study, que adultos entre 35 e 44 anos tiveram uma incidência de 38,2 por 1000 homens e 5,2 por 1000 mulheres (GULATI et al, 2020). Percebe-se que o número de casos de IAM é menor em populações abaixo de 40 ou 45 anos, correspondendo a uma porcentagem de 4 a 10\%. (JORTVEIT et al, 2020).

O novo coronavírus, causador da Síndrome Respiratória Aguda Grave Coronavírus-2 (SARS-CoV2), se tornou um problema de saúde global. Apresentando diversos sintomas clínicos, desde condições mais leves como tosse seca como também febre, diarreia, vômitos e mialgia. Entretanto, quase $20 \%$ dos pacientes diagnosticados com Covid-19 sofreram com complicações fatais. (MA, 
2020). Dentre esses pacientes mais graves, alguns eventos trombogênicos foram observados como: a liberação exacerbada de citocinas; lesão no endotélio; cascata de coagulação aumentada, apresentando um estado de hipercoagulubilidade; bloqueio da passagem de sangue; hipoxemia ocasionando insuficiência respiratória; e dispneia. (NASCIMENTO et al, 2020).

Diante do exposto, o objetivo desse trabalho foi expor um caso em que foi observado um paciente $<60$ anos de idade, sem fatores de risco, internado em Unidade de Terapia Intensiva (UTI) por complicações da Covid-19 em que umas das complicações foi IAM.

\section{RELATO DE CASO}

Paciente de 42 anos, masculino natural e procedente de Teutônia, empresário, branco.

No dia 19/05 teve confirmado Covid-19 por teste de PCR positivo, e no dia 25/05 realizou TC de tórax com comprometimento de $50 \%$ dos pulmões, caracterizada por opacificação em vidro fosco. Apresentou dispneia aos pequenos esforços, mialgia de alta intensidade, anorexia e na monitorização com oxímetro de pulso onde apresentava saturação de $89 \%$ em ar ambiente. Interna na Unidade de terapia Intensiva no dia 26/05/21, por hipoxemia e necessidade de suplementação de $\mathrm{O} 2$ com máscara de Hudson a $7 \mathrm{~L} / \mathrm{min}$. Também foi prescrito enoxaparina (40 $\mathrm{mg} / 0,4 \mathrm{ml}$ de $12 \mathrm{em} 12$ horas). No mesmo dia devido saturação manter-se abaixo de $90 \%$, aumentou-se a oferta de 02 para 15L/min por máscara de Hudson.

Equipe da UTI, opta por não realizar entubação orotraqueal, pois o paciente respondeu à suplementação de $\mathrm{O}_{2}$, com níveis de $\mathrm{PaO}_{2} 113 \mathrm{mmHg}$.

Subitamente paciente refere desconforto respiratório, queda de pressão arterial (70/40) e à cardioscopia apresentava supra de ST em derivações anteriores (V5 e V6). 
Figura 6 Monitor dos sinais vitais do paciente

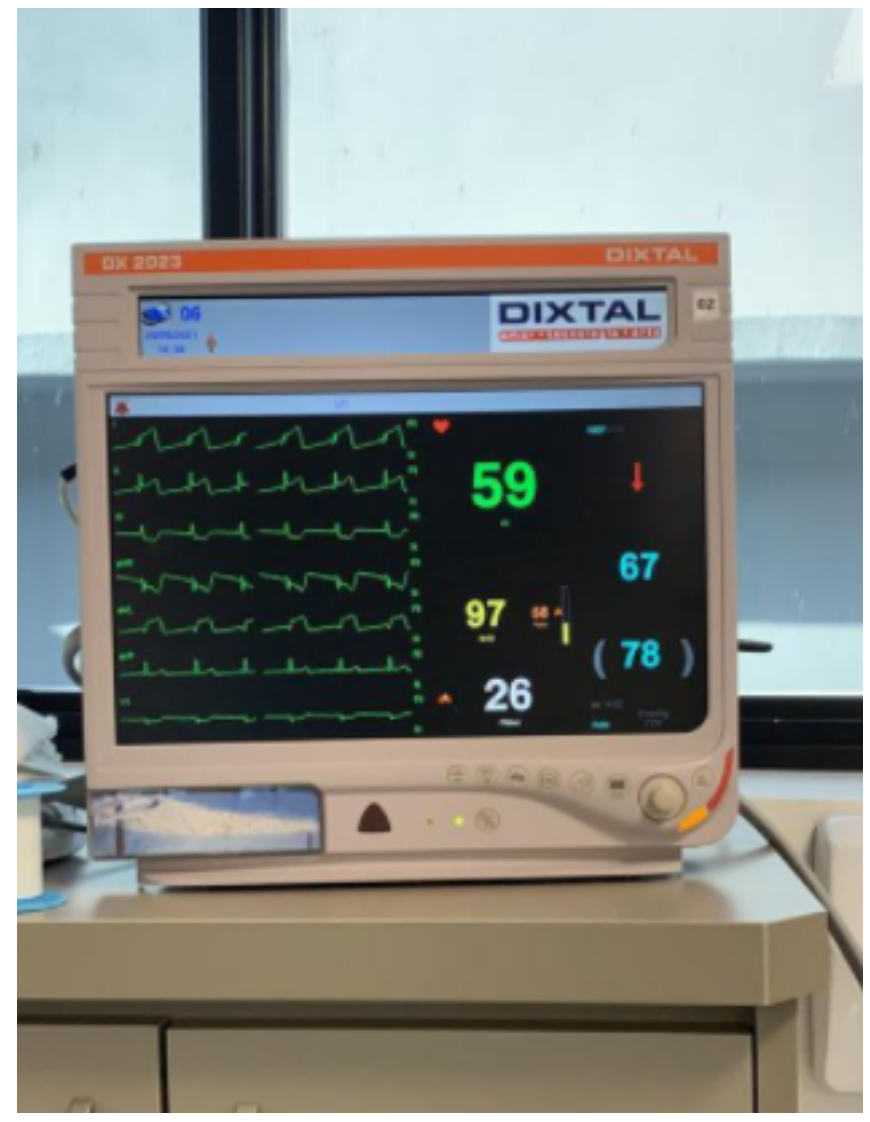

Fonte: Acervo pessoal

Foi traçado em ECG que confirmou supra de ST em V1, V2, V3, V4, V5 e V6 (Figura 7)

Figura 7. Eletrocardiograma antes da trombólise realizado dia 29/05/21 às $13 \mathrm{~h}$

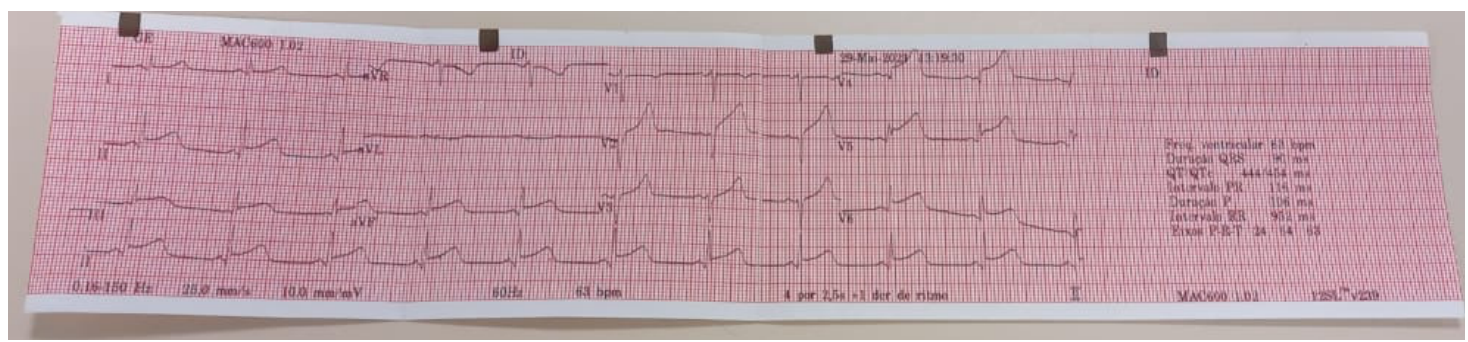

Fonte: Acervo pessoal 
Devido instabilidade hemodinâmica (queda de PA e rebaixamento do sensório) opta-se por entubação orotraqueal, com uso das seguintes drogas: etomidato $(20 \mathrm{mg})$, fentanila $200(\mu \mathrm{g})$, suxametônio (100 mg).

Entra-se em contato com serviço de hemodinâmica de referência, que propôs uso de trombolítico alteplase $15 \mathrm{mg}$ em 2 minutos, $50 \mathrm{mg}$ em 30 minutos e $35 \mathrm{mg}$ em 60 minutos, pois paciente estava dentro da janela de utilização do fármaco. Também foi administrado ácido acetilsalicílico $200 \mathrm{mg}$, clopidogrel $300 \mathrm{mg}$ e rosuvastatina $40 \mathrm{mg}$.

Não houve sucesso com a trombólise química visto pelo ECG realizado após uso do trombolítico. (Figura 8 )

Figura 8. Eletrocardiograma pós trombólise realizado dia 29/05/21 às 16h

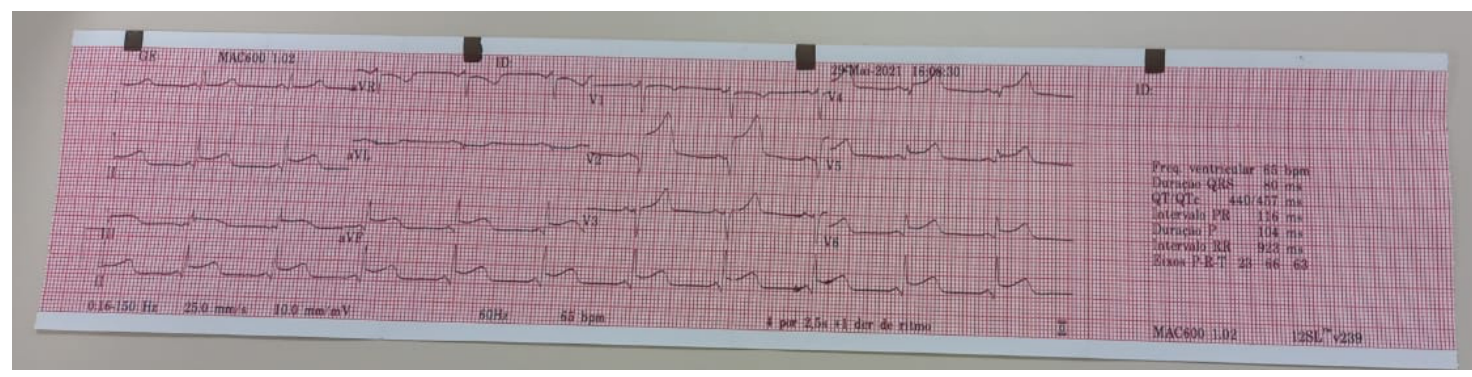

Fonte: Acervo pessoal

Paciente foi encaminhado ao serviço de hemodinâmica para realização de angioplastia primária.

No cateterismo cardíaco, o ventrículo esquerdo apresentava aumento da pressão diastólica final (PD2VE), compatível com aumento de pré-carga. Não foi realizada ventriculografia esquerda no ventrículo esquerdo. As válvulas apresentavam gradiente VE-Ao-8mmHg. O tronco da coronária esquerda (TCE) mostrava-se calibroso, bifurcado e integro. A coronária descendente anterior apresentava oclusão aguda (fluxo TIMI 0) a partir do terço médio, logo após emitir o segundo ramo diagonal. O primeiro ramo diagonal mostrava-se fino, curto e sem lesões obstrutivas significativas. O segundo ramo diagonal mostrava-se de moderado calibre, moderada extensão e sem lesões obstrutivas significativas. A 
coronária circunflexa e seus ramos não apresentavam lesões obstrutivas. A coronária direita e seus ramos apresentavam característica dominante, com calibre e fluxo angiográfico normais. A conclusão do laudo do cateterismo cardíaco foi os seguintes achados: aumento da pré-carga do ventrículo esquerdo; gradiente VEAo=8mmHg; coronária descendente anterior ocluída; e dominância coronariana direita (Figura 9).

Figura 9. Angiografia com artéria coronária obstruída

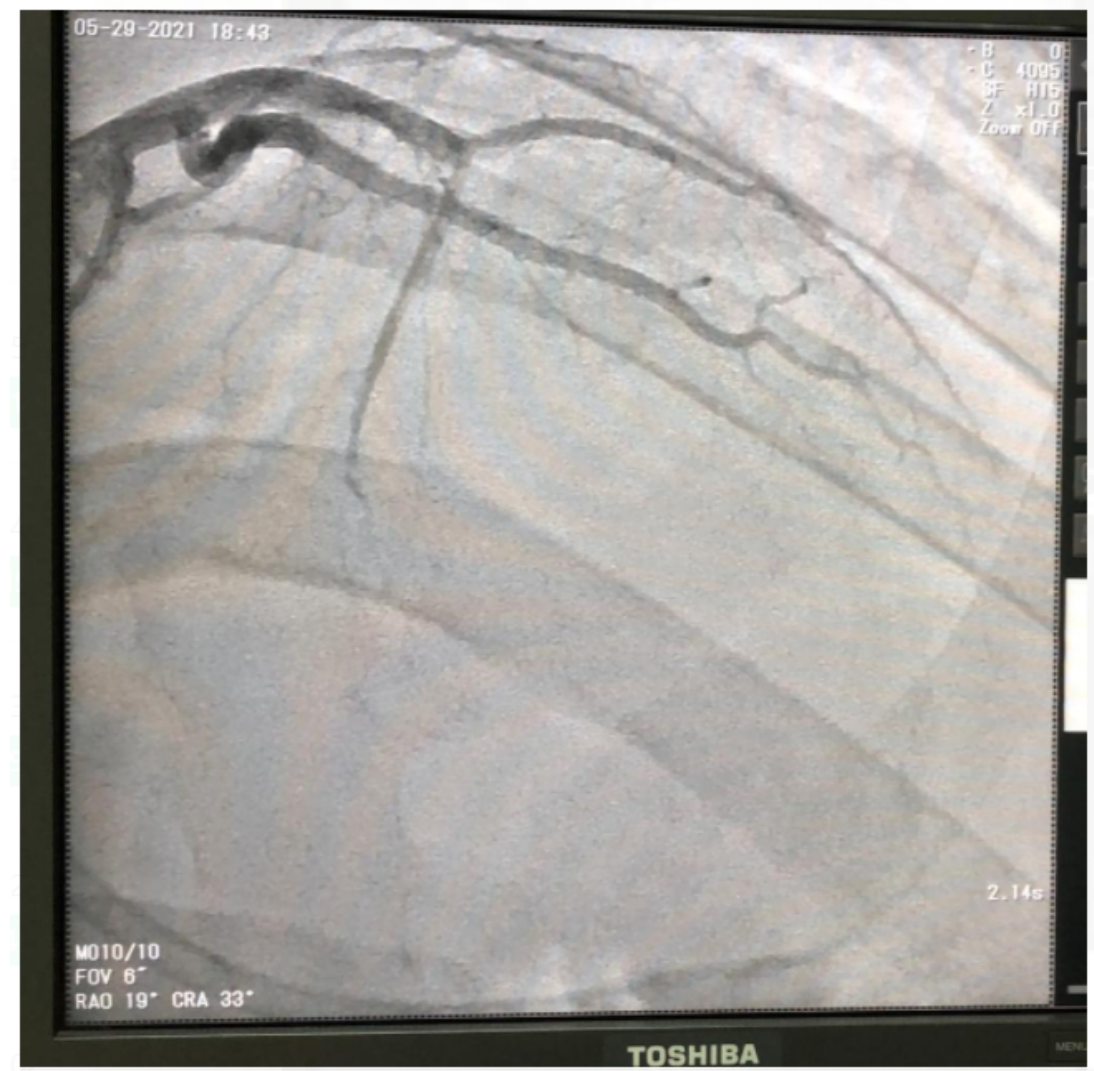

Fonte: Acervo pessoal

Com o diagnóstico procedeu-se a aspiração do trombo pelo hemodinamicista. A angioplastia coronária de resgate da coronária descendente anterior com recanalização, aspiração manual de trombos e implante de um stent farmacológico foram realizados. No momento do procedimento, o paciente se encontrava em ventilação mecânica e choque cardiogênico. Pela via radial direita, foi colocado introdutor valvulado $6 \mathrm{~F}$ e cateterizada seletivamente a coronária esquerda com cateter-guia EBU3.5 6F. Após isso, foi posicionada guia 0,014 PT no terço distal da 
coronária descendente anterior e realizada aspiração manual de grande quantidade de trombos brancos (Figura 10) com dispositivo Export advence 6F. Foi então identificada estenose residual com trombos, sendo optado pelo implante de stent. Foi utilizado balão MiniTREK 2.0x15mm para medida da extensão da estenose residual, seguido de implante de stent farmacológico Orsiro 3.5x22mm com 12 ATM. O Fluxo final foi de TIMI 3 em todos os vasos, sem complicações angiográficas imediatas. Foi administrado Heparina 7.500 UI antes do início da intervenção e Agrastat (Tirofiban) ajustado para o peso antes do início da aspiração manual de trombos. A angioplastia coronária de resgate da coronária descendente anterior com recanalização, aspiração manual de trombos e implante de um stent farmacológico, via radial direita foi feita com sucesso (Figura 11).

Figura 10. Trombo aspirado

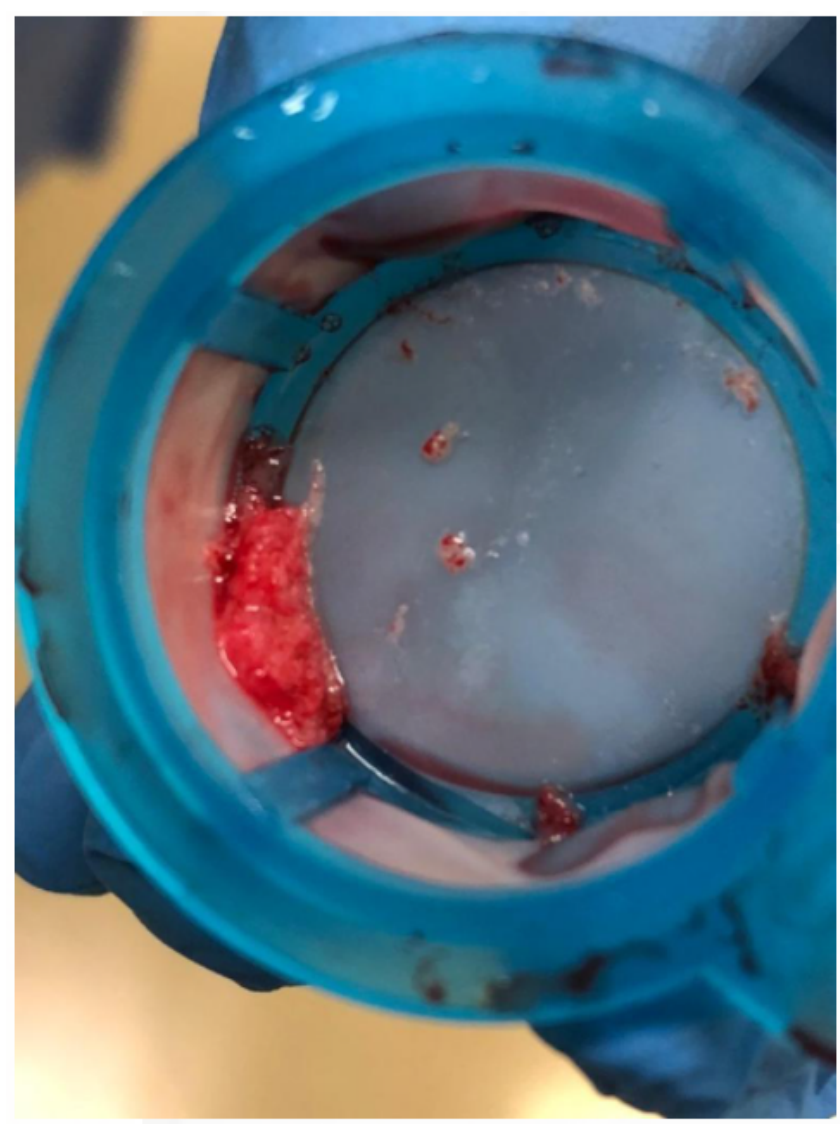

Fonte: Acervo pessoal 


\section{QUFN}

Figura 11. Angiografia pós aspiração do trombo

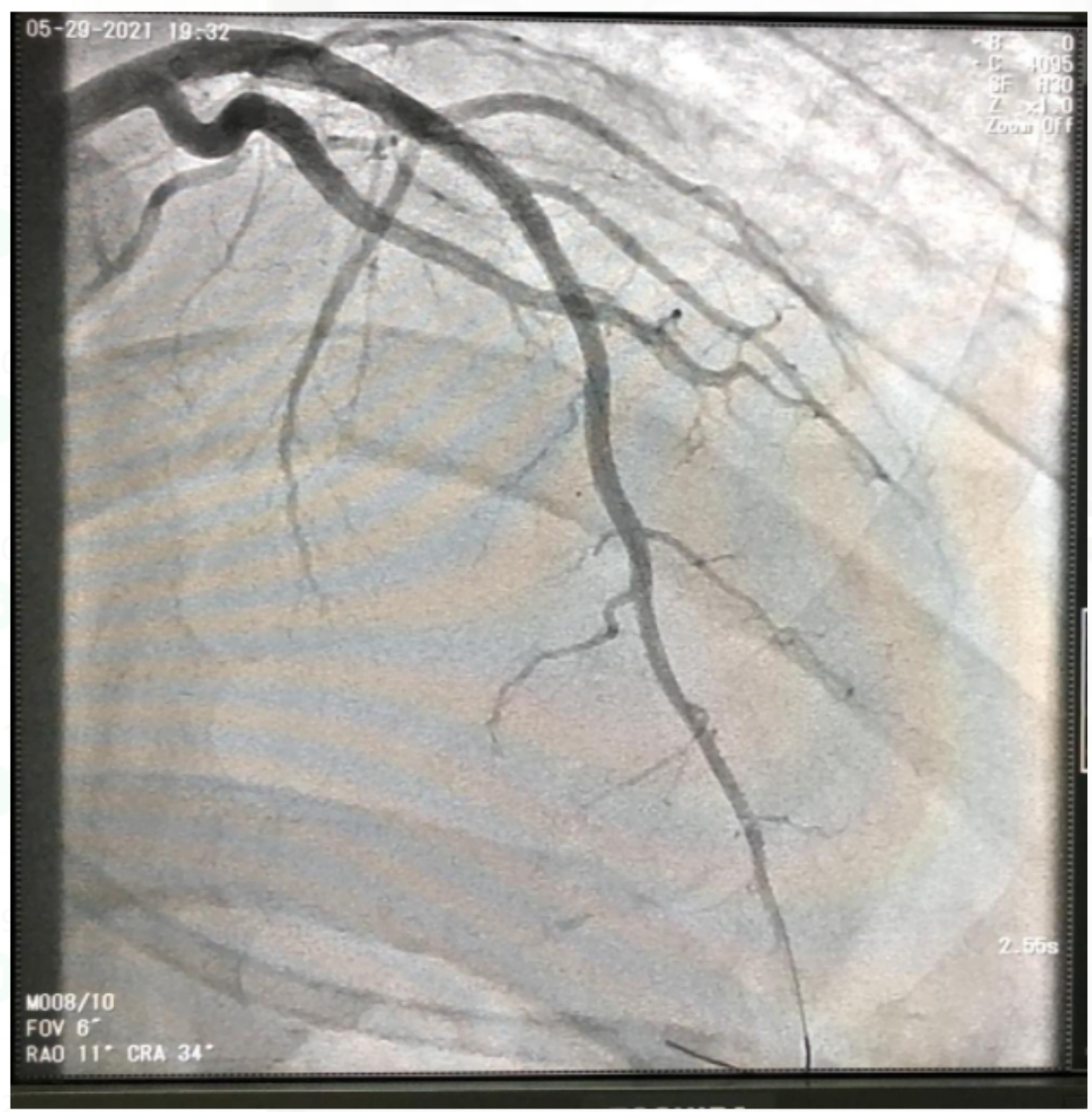

Fonte: Acervo pessoal

\section{METODOLOGIA}

Trata-se de um estudo descritivo que relata um caso ocorrido em um dos hospitais de Santa Maria - RS, no ano de 2021. Utilizou-se a base de dados eletrônica: artigos nas plataformas Scielo e Pubmed além de análises nas diretrizes mais recentes nacionais e internacionais de condutas para diagnóstico e tratamento para o infarto agudo do miocárdio em pacientes no período trans e pós diagnóstico de infecção com Covid-19. Foi realizada a revisão de prontuário, para ilustrar de forma sucinta e clara um episódio de IAM em paciente internado na UTI Covid, em um hospital na cidade Santa Maria -RS. 


\section{QUFN}

\section{RESULTADOS E DISCUSSÕES}

Analisando o caso relatado, foi possível verificar uma das consequências causada pela Covid-19. A hipercoagulabilidade causada em pacientes que mesmo não tendo idade avançada mostra-se de grande preocupação e deve ser avaliada mesmo em pacientes sem comorbidades prévias. Desse modo, deve-se haver uma equipe preparada para diagnosticar precocemente casos em que pacientes internados por Covid-19 sofrem IAM e também capaz de exercer a conduta correta em tais casos.

\section{CONCLUSÃO}

No contexto de pandemia de Covid-19, devido ao fato de haver relatos na literatura que apontam para aumento na prevalência de IAM, é preciso estar atento, e considerar esta patologia nos pacientes acometidos com Covid-19 para proceder mais rapidamente com o tratamento afim de melhorar o desfecho deste aspecto da pandemia.

\section{REFERÊNCIAS}

GULATI, R. et al. Acute Myocardial Infarction in Young Individuals. Mayo Clin Proc, v. 95, n. 1, p. 136-156, 2020.

JORTVEIT J, et al. Incidence, risk factors and outcome of young patients with myocardial infarction. Heart, 2020.

MA, H. Pathogenesis and Treatment Strategies of COVID-19-Related Hypercoagulant and Thrombotic Complications. Clin Appl Thromb Hemost, v. 26, p. 1-5, 2020.

Morgan \& Mikhail. Anestesiologia Clínica. 5. ed. Rio de Janeiro: Livraria Editora Revinter Ltda, 2017. 
Nascimento, J. H. P. et al. COVID-19 e Estado de Hipercoagulabilidade: Uma Nova Perspectiva Terapêutica. Arq. Bras. Cardiol, v.114, n. 5, p. 829-833, 2020.

SILBERNAGL, S. Fisiopatologia: Texto e Atlas. 2. ed. Porto Alegre: Artmed, 2016.

MORGAN, G. E.; MIKHAIL, M. S.; MURRAY, M. J. Clinical Anesthesiology. 6. ed. New York: Lange Medical Books/McGraw Hill Medical Pub, 2018. 